\title{
Can PET/CT detect recurrence in post- operative colorectal carcinoma patients with elevated CEA level?
}

\author{
Samar R. Ragheb ${ }^{*}$ and Sherine M. Sharara
}

\begin{abstract}
Background: PET is considered to be the most sensitive and specific modality for the detection of recurrent colorectal cancer. This study is to assess the role of F18-FDG PET/CT in post-operative assessment in a patient with colorectal cancer with elevated CEA level to rule out local recurrence and/or metastasis and hence guiding the clinician to the proper management strategy.

Results: This study was performed on 45 patients who underwent surgical resection of the colon and/or rectal cancer; they were referred for PET/CT assessment at least 3 months after surgical resection. F18-FDG PET/CT sensitivity and specificity in detecting the recurrence and/or metastasis were $96.9 \%$ and $83.3 \%$, respectively. The positive predictive value was $94.2 \%$ while the negative predictive value was $90 \%$.

Conclusion: For post-operative detection of loco-regional recurrence and metastasis in patients with colorectal cancer and elevated CEA level, 18F-FDG PET/CT can be considered as an efficient diagnostic imaging tool due to its high sensitivity and specificity which extensively affect further management.
\end{abstract}

Keywords: Colorectal cancer, 18F-FDG PET/CT, Recurrence, Carcinoembryonic antigen

\section{Background}

Colorectal cancer is one of the most common cancers worldwide and associated with a high mortality rate [1]. Radical resection is still the primary therapy for colorectal carcinoma, but local recurrence and/or metastasis rate in 2 years after operation is up to $30-40 \%$ [2]. Recurrence after initial surgery is responsible not only for significant morbidity and mortality but also for impairment for quality of life [3].

Early detection and accurate post-operative staging are very helpful for designing the subsequent therapeutic scheme, for example, secondary operation, radiotherapy, and/or chemotherapy [2].

For the early detection of recurrence, there are multiple imaging modalities that can be used including computed tomography (CT), magnetic resonance imaging (MRI), and

\footnotetext{
*Correspondence: doc_mina2004@yahoo.com

Department of Radiodiagnosis, Faculty of Medicine, Ain Shams University, Cairo, Egypt
}

fluorine-18-2-fluoro-2-deoxy-D-glucose positron emission tomography (FDG-PET) [4]. Positron emission tomography (PET) is a non-invasive molecular imaging technique that can differentiate between cancer cells and noncancerous cells by detecting differences in their metabolic activities [5].

Currently, PET is combined with CT to provide both metabolic and anatomic imaging data with a single device during a single diagnostic session. When PET and CT are combined, the sensitivity and specificity of detecting recurrent colorectal cancer range from $87 \%$ to $95 \%$ and $85 \%$ to $95 \%$, respectively [6]. PET/CT is recommended for determining management and prognosis if conventional imaging cannot confirm the presence of metastatic disease [7].

PET is the most sensitive and specific modality for the detection of recurrent colorectal cancer when used for patients with increasing carcinoembryonic antigen (CEA) levels that had done non-diagnostic CT imaging [8] as we

\section{Springer Open}

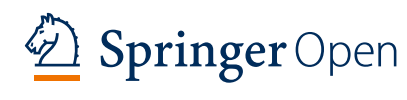

(c) The Author(s). 2020 Open Access This article is licensed under a Creative Commons Attribution 4.0 International License, which permits use, sharing, adaptation, distribution and reproduction in any medium or format, as long as you give appropriate credit to the original author(s) and the source, provide a link to the Creative Commons licence, and indicate if changes were made. The images or other third party material in this article are included in the article's Creative Commons licence, unless indicated otherwise in a credit line to the material. If material is not included in the article's Creative Commons licence and your intended use is not permitted by statutory regulation or exceeds the permitted use, you will need to obtain permission directly from the copyright holder. To view a copy of this licence, visit http://creativecommons.org/licenses/by/4.0/. 
will discuss in this study. More recently, there have been studies indicating that PET/CT may be useful in detecting recurrence in patients with normal CEA levels [9].

\section{Methods}

Our study was a retrospective analysis study carried out in the radiodiagnosis department at our university on 45 patients (17 were females and 28 were males) with known colorectal cancer undergone surgical resection (at least 3 months after surgical resection) in the period from June 2018 to June 2019. An informed consent was obtained from all patients.

The patient's age ranged from 34 to 81 years with the median age 58.7 years. The indication of the examination varied from elevated tumor markers (CEA), assessment of treatment response, routine follow-up, abnormality in other imaging modalities, or abnormal endoscopic findings. Some patients were referred for more than one cause.

Inclusion criteria:

- Patients with pathologically proven colorectal carcinoma and underwent radical resection

- No age or sex predilection

Exclusion criteria:

- Patients with blood glucose level $>390 \mathrm{mg} / \mathrm{dl}$ at the time of the study

- Patients with a history of previous hypersensitivity reaction to contrast material

- Bad general condition

- Patients with renal impairment (elevated serum creatinine $>2 \mathrm{mg} / \mathrm{dl}$ or decreased glomerular filtration rate $<30 \mathrm{mg} / \mathrm{dl}$ )

Most patients followed a post-operative follow-up program including clinical and radiological follow-up. The measurement of serial carcinoembryonic antigen (CEA) was integrated in the post-operative monitoring. Recurrence was suspected on the basis of elevated levels of CEA.

\section{$\mathrm{PET} / \mathrm{CT}$ acquisition and processing}

Combined PET/CT scan was performed using a hybrid $\mathrm{PET} / \mathrm{CT}$ system (Ingenuity, TF PET/CT/Philips, the Netherlands). The integrated CT system is a 64 multislice scanner. The acquisition of co-registered CT and PET images was performed in one session. Adequate patient preparation rules were strictly followed. Patients were instructed to fast except for glucose-free hydration for $4-6 \mathrm{~h}$ before injection of 18F-FDG. The scan was performed $40-60 \mathrm{~min}$ after IV injection of $3.7 \mathrm{MBq} / \mathrm{kg}$ (maximum dose $370 \mathrm{MBq}$ ) equivalent to $0.1 \mathrm{mCi} / \mathrm{kg}$ (maximum dose $=10 \mathrm{mCi}$ ) of 18F-FDG. The patients were examined in supine position. A whole-body examination was performed starting from the skull base to mid thighs. A PET emission scan was performed over several bed positions (5-7), each with an axial field of view of approximately $15 \mathrm{~cm}$ per bed position with an in-plane spatial resolution of $4 \mathrm{~mm}$ covering the same field of view as with CT. The acquisition time of emission data was $2 \mathrm{~min}$ per bed position in the time range that was between 13 and $17 \mathrm{~min}$.

A fully diagnostic CT scan was performed using the following parameters: $350 \mathrm{~mA}, 120 \mathrm{kV}, 0.5 \mathrm{~s}$ tube rotation time, slice thickness $5 \mathrm{~mm}, 8-\mathrm{mm}$ table feed, and $3 \mathrm{~mm}$ incremental reconstruction. A IV contrast administration $(80-120 \mathrm{ml})$ of a low-osmolarity iodinated contrast agent and negative oral contrast agent (water) for bowel was used.

All PET, CT, and PET/CT images were reconstructed and viewed on a Philips workstation, which provided multi-planar reformatted PET, CT, and fused PET/CT images with linked cursors as well as 3D maximum intensity projection (MIP).

\section{Image analysis}

A combined team, including one experienced nuclear medicine physician and one experienced radiologist (each has 10 years of experience), interpreted the PET, CT, and fused PET/CT images. The PET images were reviewed by the nuclear medicine physician. Fused PET/CT images were then analyzed separately by each of them, and each was blind to the other's findings.

\section{Interpretation of the PET/CT findings}

Visual assessment alone was adequate for interpreting PET findings as positive or negative. According to the IHP definitions, residual masses of $2 \mathrm{~cm}$ or more in greatest transverse diameter (GTD) with 18F-FDG activity visually exceeding that of mediastinal blood pool structures are considered PET-positive, whereas residual masses 1.1 to $1.9 \mathrm{~cm}$ are considered PET-positive only if their activity exceeds surrounding background activity. A smaller residual mass or a normal-sized lymph node (e.g., $<1 \times 1 \mathrm{~cm})$ should be considered positive for the disease if its activity is higher than that of the surrounding background.

Hepatic or splenic lesions should be considered as positive if their uptake is higher than or equal to that of the liver or spleen and negative if their uptake is lower than that of the liver and spleen.

Lung nodules of $5 \mathrm{~mm}$ in patients should be considered as positive if the FDG uptake is greater than the mediastinal blood pool. Metastasis cannot be excluded in lung nodules less than half a centimeter. 
If there was a clearly multifocal increase in the FDG uptake in the bone marrow, the patient was considered as PET-positive. The diffuse pattern of uptake of reactive bone marrow hyperplasia after chemotherapy can mimic or mask diffuse bone marrow involvement; therefore, appropriate history was critical. A delay of 3 to 4 weeks after completion of therapy permits the physiologic marrow activity to abate.

The existence of recurrent colorectal cancer was determined based on histopathological examination or follow-up. In the absence of a positive pathology, clinical follow-up was done for at least 12-18 months of observation either with PET/CT or conventional imaging modalities showing progression in measurable lesions by the RECIST criteria or by adequate response to therapy (some patients received radiotherapy and/or chemotherapy as well as target therapy).

\section{Results}

Recurrent colorectal cancer was suspected on the basis of an elevated level of CEA. The study included 45 patients. PET/CT was positive for recurrent colorectal cancer (either local recurrence or distant metastasis) in 34 patients $(75.5 \%)$ and negative in 11 patients $(24.5 \%)$.
Out of the 34 patients with positive PET/CT (Figs. 1, 2, 3, 4, 5 and 6), 32 were true-positive cases. Five patients had a recurrence of CRC confirmed by histopathological examination. Twenty-seven patients had recurrent CRC based on clinical and radiological follow-up associated with the measurement of CEA level, and 24 of them showed regression of the detected lesions and a decrease of CEA level after receiving appropriate treatment. Three patients showed progression in the size and activity of the detected lesions with persistent elevation of the CEA level. Serum CEA was elevated in 30 patients with positive PET/CT findings, and its level ranged between $0.6 \mathrm{ng} / \mathrm{ml}$ and $100 \mathrm{ng} / \mathrm{ml}$.

Two cases showed hypermetabolic mesenteric lymphadenopathy in PET/CT pathologically proven to be negative for malignancy (false-positive cases). Out of the eleven patients with negative PET/CT findings, ten of them proved to be true-negative cases based on the serial measurement of CEA and clinical and radiological follow-up. One patient had negative initial PET/CT and normal CEA levels with abnormal FDG accumulation in follow-up PET/CT and elevation of the CEA level (false-negative).

The sensitivity, specificity, and positive and negative predictive values were estimated using a standard statistical formula for calculating the diagnostic

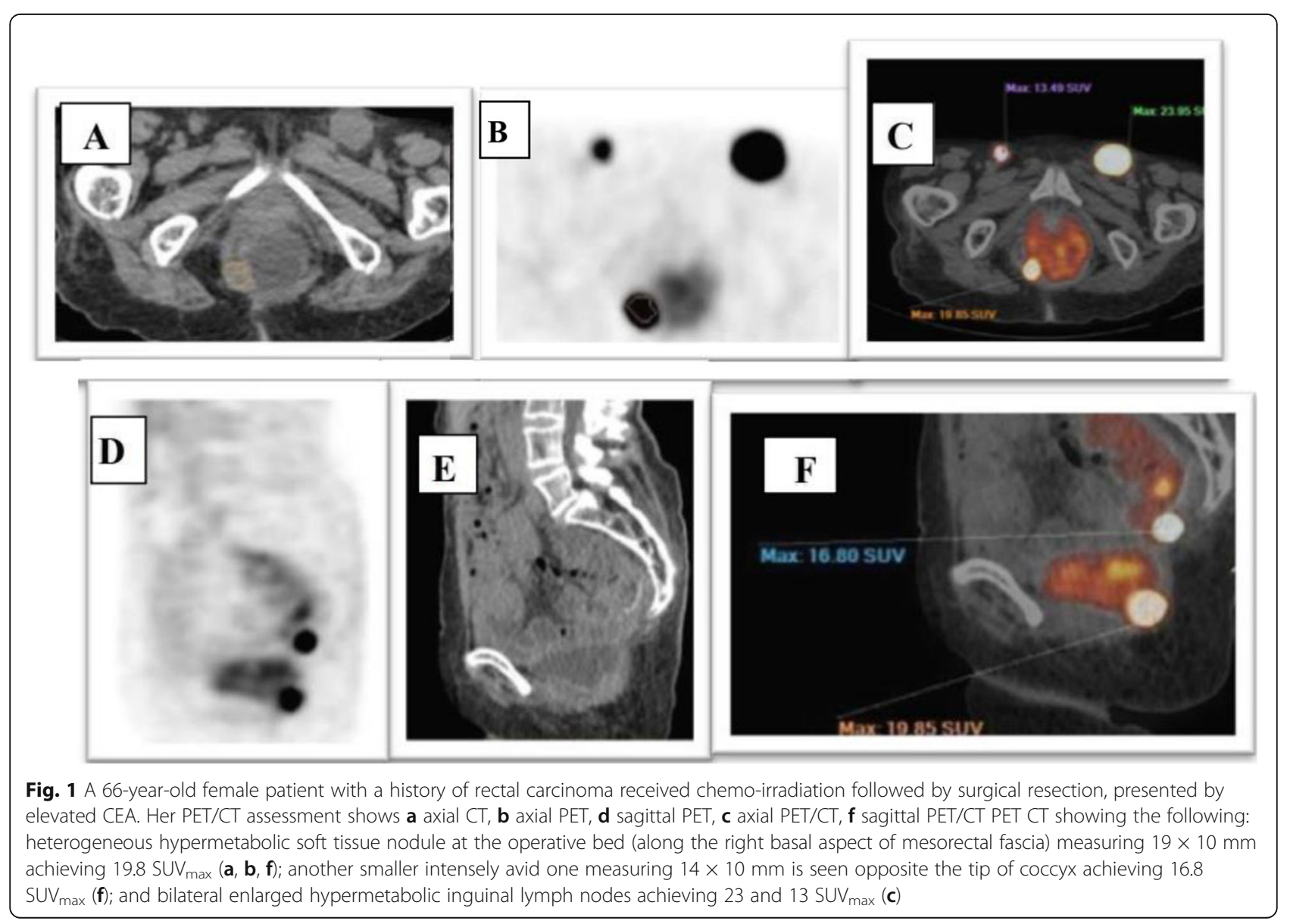


Table 1 Demonstrates the sensitivity, specificity, and positive and negative predictive values of 18F-FDG PET/CT in detecting recurrent colorectal cancer

\begin{tabular}{llll}
\hline Sensitivity & Specificity & Positive predictive value & Negative predictive value \\
\hline $96.9 \%$ & $83.3 \%$ & $94.2 \%$ & $90 \%$ \\
\hline
\end{tabular}

efficacy of PET/CT in detecting recurrent colorectal cancer (Table 1).

Comparisons between CECT and PET/CT findings were done using crosstabs and kappa value as well as confidence interval 99\% (Table 2).

\section{Discussion}

Highlighting on postoperative patients with colorectal carcinoma is to detect whether recurrence and metastasis exist or not, which can be detected by traditional imaging only when lesion sites present morphological changes. But it is difficult to distinguish early recurrence and metastasis from the inflammatory process and anatomic changes caused by operation and radiotherapy. A quarter of the recurrent colorectal carcinoma confines to the original operative site, and these patients can be cured by a second operation. The only way to reduce metastasis is early detection of recurrence. 18F-FDG PET shows metabolism changes that happen before morphological changes, which makes it detect recurrence earlier than conventional imaging [2].

In the current study, we evaluated 45 patients for the presence of recurrence and/metastasis. According to the recommendations of ASCO, carcinoembryonic antigen is considered the best marker for monitoring metastatic colorectal cancer, and its measurement can be useful in the detection of asymptomatic recurrences. After surgical resection, it was found that the tumor has recurred in almost $90 \%$ of patients who have elevated postoperative CEA levels [10]. In the current study, 30 patients had elevated serum CEA levels. The sensitivity, specificity, and positive and negative predictive values of FDG $\mathrm{PET} / \mathrm{CT}$ in detecting CRC recurrence/metastasis in patients with elevated serum CEA as the following: 96.9\%, $83.3 \%, 94.2 \%$, and $90 \%$. That was in accordance with Metser et al. [10] who reported that the sensitivity ranges from $79-100 \%$ and the specificity ranges from $50-100 \%$. The results of the current study fall within this range, so recurrent CRC was detected by FDG PET/
CT with high sensitivity and specificity (Figs. 2, 3, 4, 5, and 6).

Agarwal et al. [9] reported that FDG PET/CT is more sensitive than conventional imaging in the evaluation for recurrence of CRC patients with rising CEA levels. In the current study, we also concluded that PET/CT is useful in the detection of recurrence in patients with normal CEA values but with suspicious clinical symptoms or radiologic findings. In the current study, two patients had normal serum CEA levels with positive PET/CT findings.

The diagnosis of local recurrence and differentiating it from post-operative and/or radiotherapy changes constitute a diagnostic challenge for conventional imaging as these changes may appear on CT images for many years and be indistinguishable from tumor recurrence [11]. In the current study, the detection of local recurrence by $\mathrm{PET} /$ contrast-enhanced CT was higher compared with enhanced CT. Ten lesions were detected as local recurrence and/or operative bed findings by CECT while PET/ CT detect 17 lesions. So, PET/CT revealed additional information by detecting occult malignant lesions which were not detected in CECT. These results agree with Kitajima et al. [4] and Chiewvit et al. [12] which showed that the sensitivity of detecting local recurrence by PET/ contrast-enhanced $\mathrm{CT}$ was relatively high compared with enhanced CT (96.7 vs 80.0\%). Also, the study done by the study of Kitajima et al. [11] demonstrated that FDG-PET/ CT seems to be the method of choice in the evaluation of presacral fibrotic tissue in patients who have undergone rectal amputation [12]. In the current study, out of the 16 patients diagnosed with recurrence, PET/CT detected 4 patients with pararectal soft tissue masses and 3 patients with presacral soft tissue lesions.

Liver metastasis is very common in patients with colorectal cancer with an incidence of approximately 50$60 \%$ [11]. The presence of hepatic metastasis is the main determinant of survival and guides the therapeutic strategy, particularly in patients with colorectal carcinoma [13]. In the current study, 12 patients proved to have

Table 2 Demonstrates the number of lesions detected in CECT and PET/CT in different sites

\begin{tabular}{llllll}
\hline & CT no. of lesions & PET/CT no. of lesions & Agreement & Kappa value & Confidence interval 99\% \\
\hline Local recurrence & 10 & 17 & $(61.1 \%)$ & 0.147 & $0.437-0.462$ \\
LN metastasis & 38 & 58 & $(63.5 \%)$ & 0.112 & $0.123-0.140$ \\
Hepatic deposits & 50 & 65 & $(82.5 \%)$ & 0.013 & $0.013-0.029$ \\
Peritoneal deposits & 9 & 14 & $(76.5 \%)$ & 0.469 & $0.048-0.059$ \\
Osseous deposits & 6 & 13 & $(46.2 \%)$ & 0.646 & $0.005-0.009$ \\
\hline
\end{tabular}




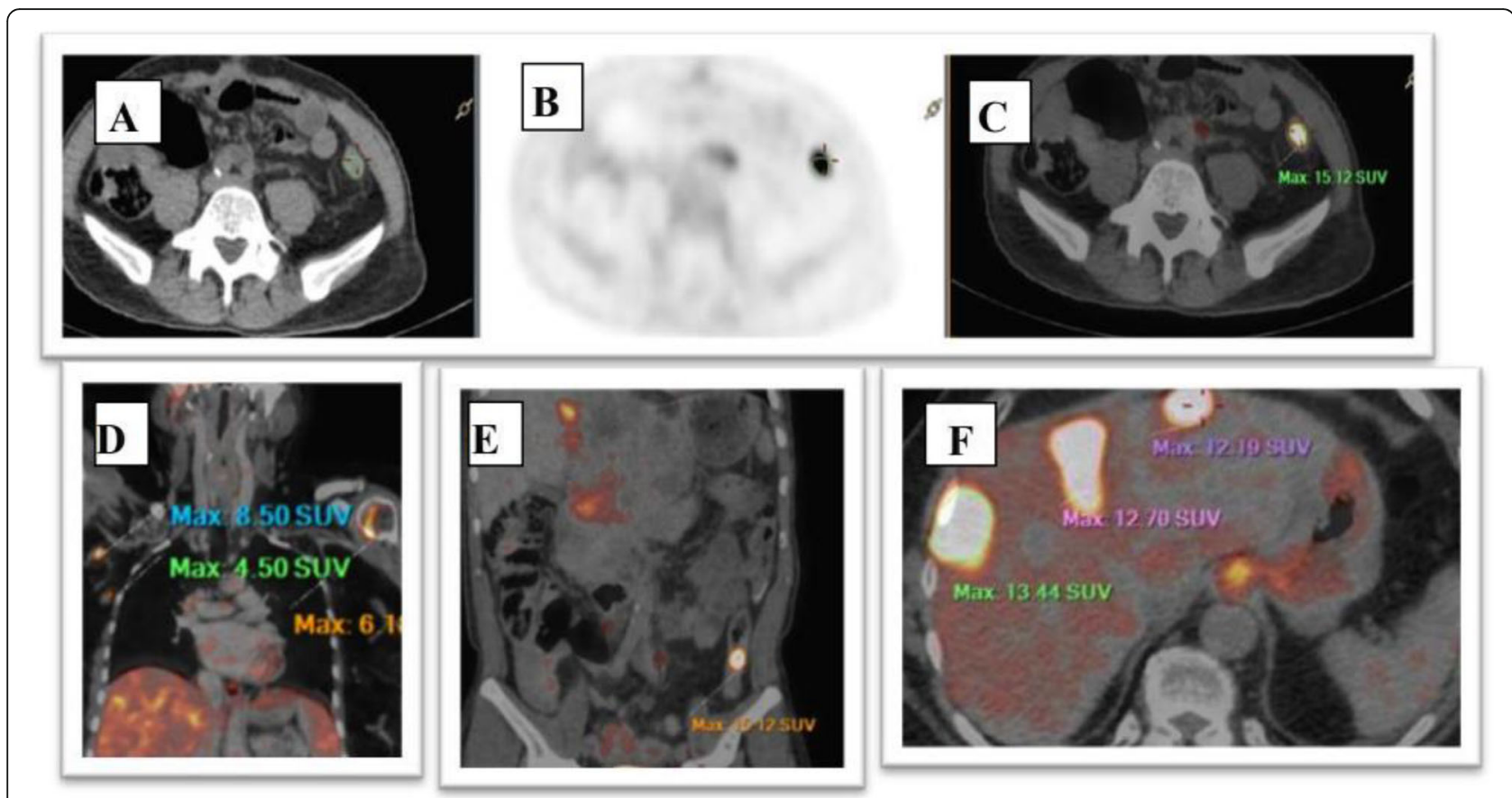

Fig. 2 A 75-year-old male patient with a history of colon cancer treated by surgical resection. He was referred for routine follow-up by PET/CT. a Axial CT, $\mathbf{b}$ axial PET, $\mathbf{c}$, e axial PET/CT, $\mathbf{d}$, e coronal PET/CT showing the following: focal mural increased FDG uptake is noted at the distal aspect of the descending colon, just below the surgical anastomotic site achieving $15.12 \mathrm{SUV}_{\max }$ proved to be recurrent by endoscopy and histopathology $(\mathbf{a}, \mathbf{b}, \mathbf{c})$; multiple hypermetabolic metastatic hepatic focal lesions achieving SUV max $_{\text {up }}$ to 13.44 (f); and multiple hypermetabolic focal osseous deposits, achieving SUV max of 8.50 (d)

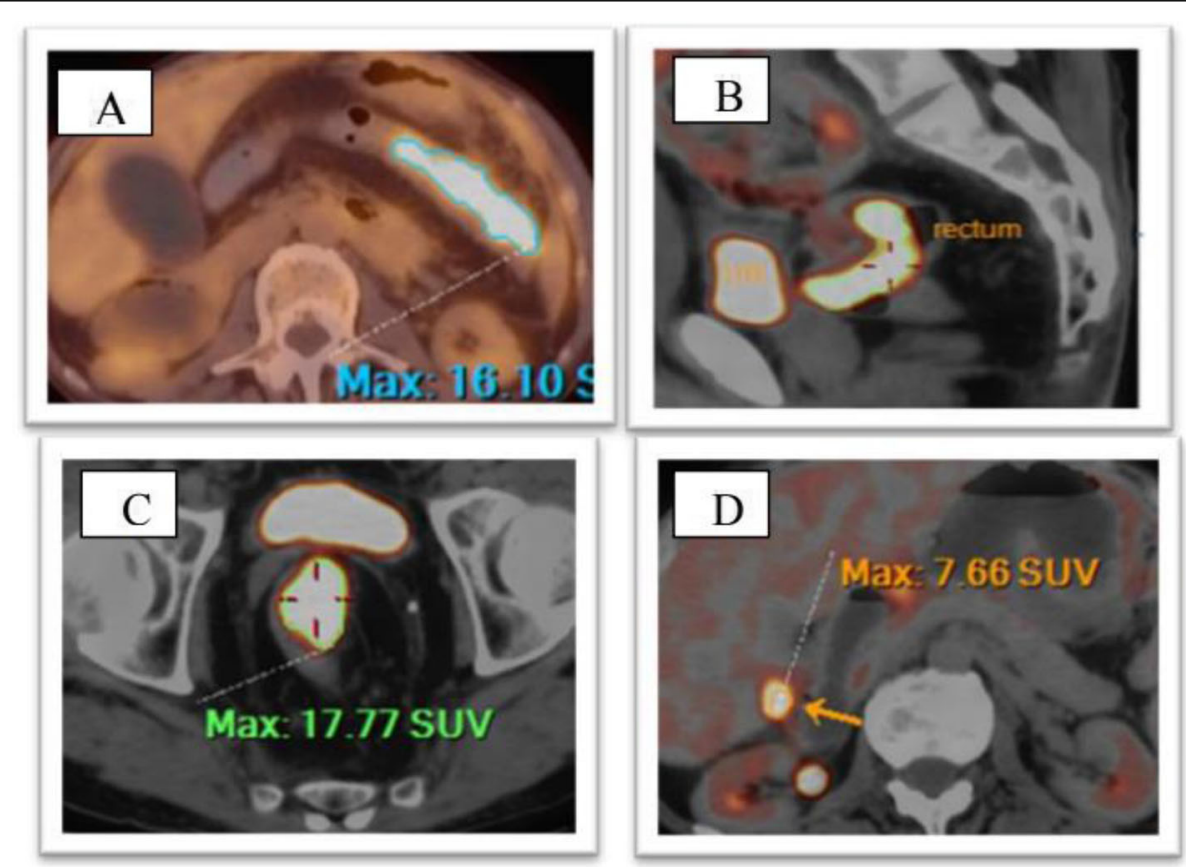

Fig. 3 A 61-year-old male patient with a history of colon cancer treated by surgical resection. He was referred for PET/CT assessment due to elevated CEA. a, c, d Axial PET/CT cuts through the abdomen and the pelvis, $\mathbf{b}$ sagittal PET/CT through the pelvis showing the following: irregular thickening of the wall of distal transverse colon with hypermetabolic activity reaching up to 16 SUV $V_{\max }$ proved to be malignant on endoscopy and biopsy (a); irregular mucosal mass at the rectum projecting into its lumen with hypermetabolic activity reaching up to 17.7 SUV $V_{\max }(\mathbf{b}, \mathbf{c})$; and the liver showing a small hypermetabolic focal lesion measuring $1 \mathrm{~cm}$ and achieving $7.6 \operatorname{SUV}_{\max }(\mathbf{d})$ 


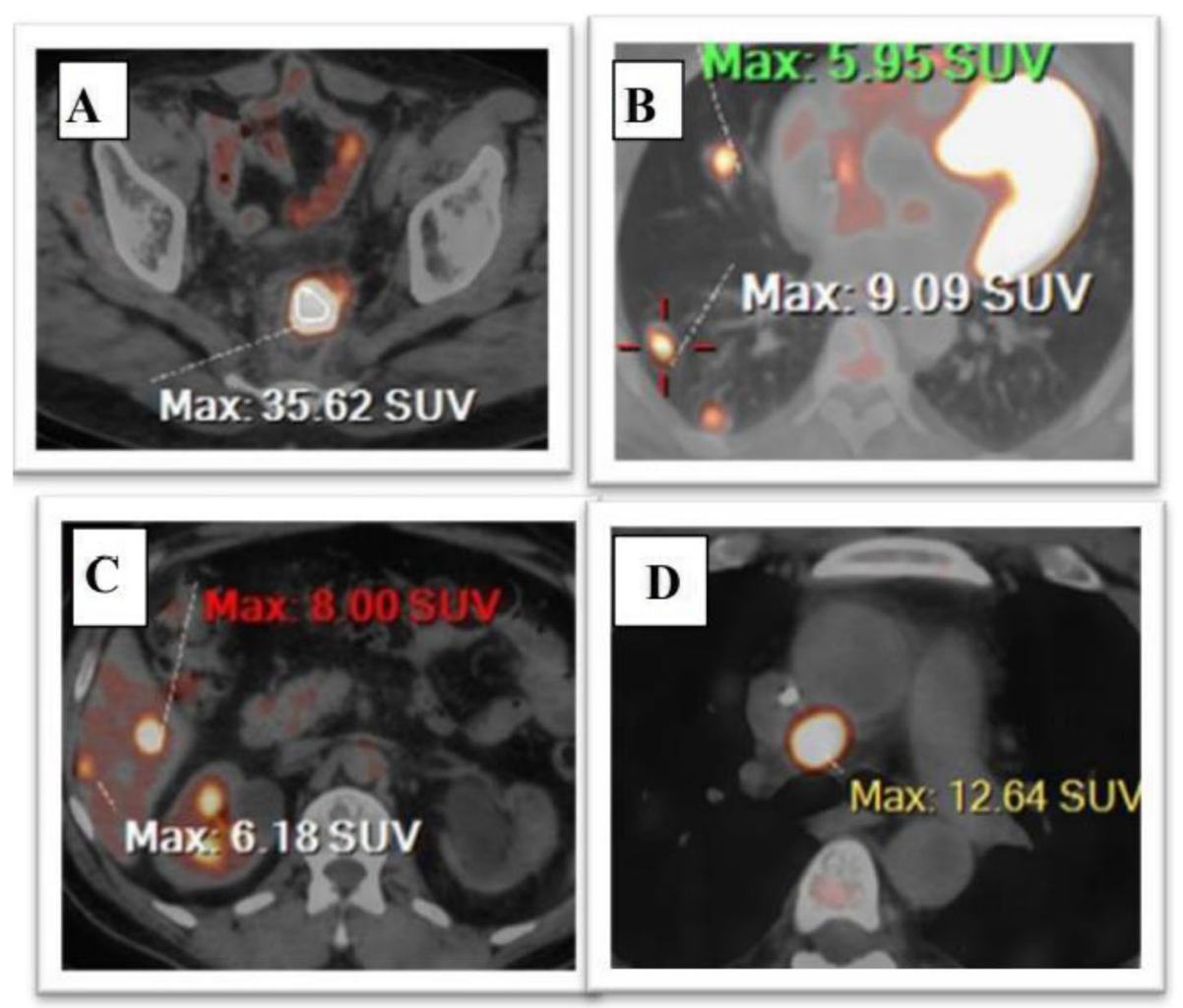

Fig. 4 A 55-year-old male with a surgically resected rectal cancer, presented by elevated CEA and was referred for PET/CT assessment. a, b, c, d Axial PET/CT images through the pelvis, chest, and abdomen showing the following: left pararectal hypermetabolic soft tissue lesion achieving 35.62 SUV $\max (\mathbf{a})$; multiple hypermetabolic metastatic pulmonary deposits, the most active of them achieved $9.09 \mathrm{SUV}$ max $(\mathbf{b})$ associated with hypermetabolic mediastinal lymph nodes, the most active of them achieve 12.64 SUV max (d); multiple hypermetabolic metastatic hepatic focal lesions, the most active of them achieve $8 \operatorname{SUV}_{\max }(\mathbf{c})$

metastatic hepatic lesions with higher detection rate of PET/CT over CECT in the detection of metastatic hepatic deposits. Fifty lesions were detected by CT and 65 lesions were detected by PET/CT which detected occult metastatic hepatic focal lesions. The superiority of PET/ CT over CECT owes to the latter's inability to diagnose small masses (less than $15 \mathrm{~mm}$ in diameter) as benign or malignant. These results are comparable to those of Ali and Abd Elkhalek [14] and Agarwal et al. [9] which demonstrated that FDG PET is highly sensitive for the detection of liver metastases, and the routine FDG-PET/CT assessment of patients with hepatic metastasis has a significant impact on disease staging and selection of candidates suitable for solitary liver metastasis resection.

PET/CT could also be used in monitoring the treatment efficacy of local therapy of isolated lung and liver metastatic lesions. The local therapy approaches of hepatic metastasis are radiofrequency ablation (RFA), transcatheter arterial chemoembolization (TACE), and cryoablation. Traditional imaging techniques could not discriminate between tissue edema and tumor remnant. However, PET provides a functional imaging of the levels of metabolism within the suspected foci and also the whole body, so it substantially helps the clinicians in recognizing tumor remnants [15]. In the current study, one patient underwent radiofrequency ablation for hepatic metastasis which showed successful ablation of the focal hepatic lesion with no residual FDG uptake. This is also comparable with the results of Veit el al [16]. which showed that PET/CT plays a distinctive role in the follow-up of patients undergoing RFA of the liver lesions.

In the current study, eight patients proved to have metastatic peritoneal deposits. The number of detected lesions by CECT was 9, and 14 lesions were detected by PET/CT which showed the superiority of PET/CT over CECT which detected occult peritoneal deposits.

Lymph node metastasis is an important prognostic factor in patients with colorectal cancer [17]. In the current study, 17 patients proved to have metastatic lymph nodes with higher detection rate by PET/CT over CECT as regards the number of the detected lesions. Thirtyeight lymph nodes were detected by CECT and 58 lymph nodes were detected by PET/CT which detected 18F-FDG avid subcentimetric lymph nodes; these additional sites of metastatic involvement would have been missed if assessment was done using CT alone, and this 


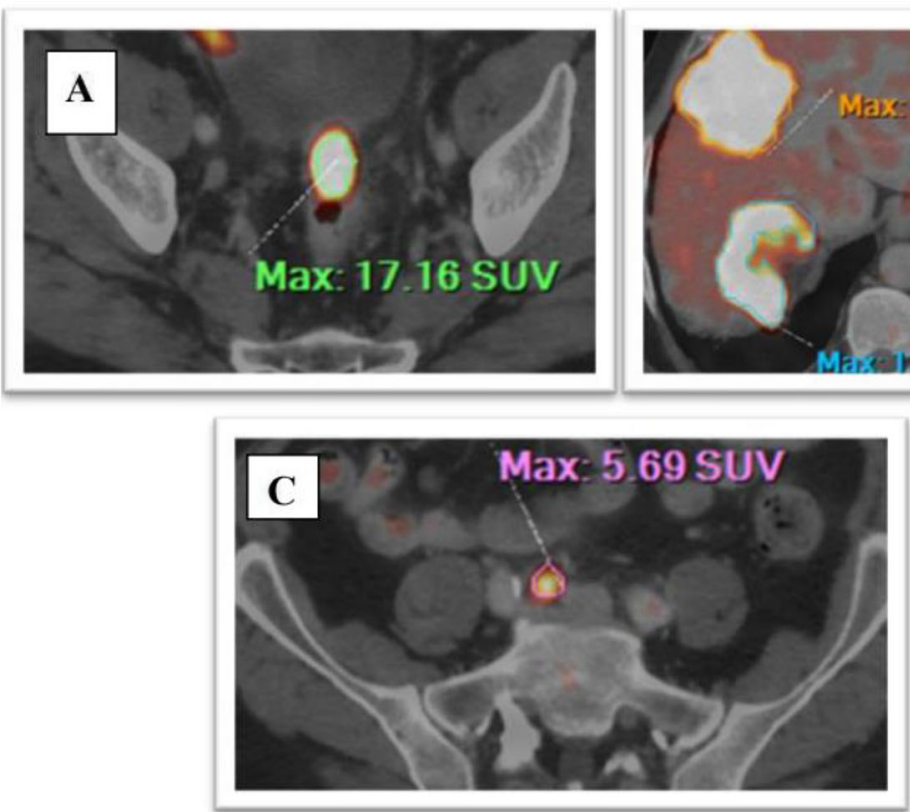

Fig. 5 A 65-year-old male patient with a history of rectal cancer treated first surgically followed by chemotherapy. He was presented with elevated CEA and referred for PET/CT assessment. $\mathbf{a}, \mathbf{b}, \mathbf{c}$ Axial PET/CT images through the abdomen and the pelvis showing the following: recurrent hypermetabolic soft tissue nodule at the pelvic operative bed achieving activity about $17.16 \mathrm{SUV}_{\max }(\mathbf{a})$; hypermetabolic hepatic focal lesions achieving about 13 and 13.7 SUV $_{\max }(\mathbf{b})$; hypermetabolic right internal iliac lymph nodes achieving activity about 5.69 SUV $\max$ (c)

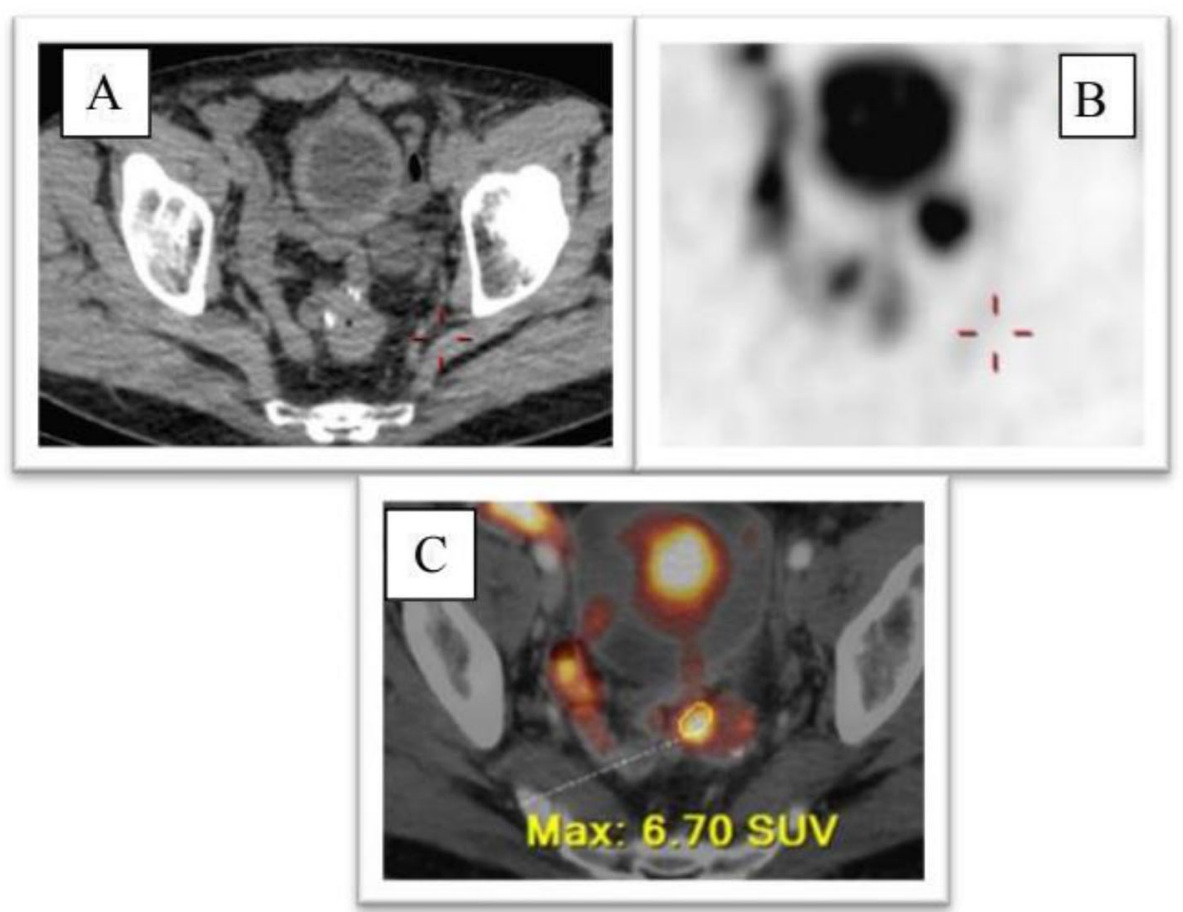

Fig. 6 A 70-year-old male patient with a history of surgically resected sigmoid cancer followed by chemotherapy, presented by elevated CEA and referred for PET/CT assessment. a Axial CT, $\mathbf{b}$ axial PET, and $\mathbf{c}$ axial PET/CT showing the following: ill-defined metabolically active soft tissue lesion at the left side of the pelvis related to the recto-sigmoid anastomotic site measuring $29 \times 19 \mathrm{~mm}(\mathbf{a}$, b) and achieving 6.70 SUV $\max (\mathbf{c})$ 
result in up-staging and consequently change the management plan in these patients. CT's strengths are its accurate depiction of anatomic abnormalities and its ability to define structures that are below the resolution of conventional nuclear medicine. A major limitation of CT however is its reliance on anatomic criteria in order to identify the pathological condition, for example using a 1-cm short axis dimension threshold for pathological lymphadenopathy.

However, lymph nodes smaller than $1 \mathrm{~cm}$ can contain malignant cells and, in addition to reactive or inflammatory processes, can result in nodes larger than $1 \mathrm{~cm} \mathrm{[1].}$

Nodal metabolic activity assessment using FDG PET is not directly reliant on the nodal size to determine the presence or absence of malignancy. Nodes that are not enlarged can contain tumor on FDG PET. However, PET can give false-positive results due to inflammation. In addition, some cystic or mucinous nodal metastasis may not show significant FDG uptake [18].

Bone metastasis from colorectal carcinoma is relatively uncommon [1]. In the current study, patients had metastatic bone deposits with higher detection rate by PET/ CT over CECT. Six lesions were detected by CECT and 13 were detected by PET/CT with retrospective detection of 7 occult lesions missed on CT. That was in accordance with Ali and Abdelkhalek [19].

PET scanning can identify osseous metastases at an early stage of growth before host reactions to tumor cells occur (active osseous deposits without structural abnormalities) [18]. Early malignant bone marrow infiltration can be depicted by 18F-FDG PET because of the very early increased metabolism of glucose in the neoplastic cells. The combined PET/CT is unique as it can scan the whole body in one session, and it can provide us with the functional and anatomic data in co-registered images. Combined PET/CT improved the CT ability to detect and characterization of metastatic osseous deposits, which is essential for proper staging and further management planning [19].

The overall comparison between CECT and PET/CT in the current study showed significant superiority of PET/CT over CECT.

The limitations of our study were as follows:

- Relatively small number of the studied population.

- Patient referral bias and intra- and inter-observer variation for conventional imaging interpretation influenced the selection of eligible patients.

\section{Conclusion}

For post-operative detection of loco-regional recurrence and metastasis in patients with colorectal cancer and elevated CEA level, 18F-FDG PET/CT can be considered as an efficient diagnostic imaging tool due to its high sensitivity and specificity which extensively affect further management.

\section{Abbreviations}

18F-FDG: 18F-Fluorodeoxyglucose; ASCO: American Society of Clinical Oncology; CEA: Carcinoembryonic antigen; CRC: Colorectal cancer; CT: Computed tomography; CECT: Contrast-enhanced computed tomography; GTD: Greatest transverse diameter; IHP: International Harmonization Project; IV: Intravenous; MRI: Magnetic resonance imaging; MIP: Maximum intensity projection; PET/CT: Positron emission tomography/ computed tomography; RFA: Radiofrequency ablation; RECIST: Response Evaluation Criteria in Solid Tumors; SUV max: Maximum standardized uptake value; TACE: Transcatheter arterial chemoembolization

\section{Acknowledgements \\ Not applicable}

Authors' contributions

SR and SS contributed equally to this work. The authors have read and approved the final manuscript.

\section{Funding}

The author states that this work has not received any funding.

\section{Availability of data and materials}

The datasets used and/or analyzed during the current study available from the corresponding author on reasonable request.

\section{Ethics approval and consent to participate}

This study was approved by the ethical committee of Ain Shams University on June 2018 (no reference number was given at that time). All patients included in this study gave written informed consent to participate in this research by the patients themselves or by primary degree relatives.

\section{Consent for publication}

All patients included in this research gave written informed consent to publish the data contained within this study.

\section{Competing interests}

The author declares that she has no competing interests.

Received: 23 November 2019 Accepted: 17 March 2020

Published online: 27 March 2020

\section{References}

1. Cinar A, Esra A, Meliha K et al (2013) Restaging of colorectal cancer and PET/CT. Turk J Surg 29:76-80

2. Han A, Xue J, Zhu D et al (2011) Clinical value of 18F-FDG PET/CT in post operative monitoring for patients with colorectal carcinoma. Cancer Epidemiol 35:497-500

3. Shaefer O, Langer M (2007) Detection of recurrent rectal cancer with CT, MRI and PET/CT. Eur Radiol 17:2044-2054

4. Kitajima K, Murakami K, Yamasaki E et al (2009) Performance of integrated FDG PET/ contrast-enhanced CT in the diagnosis of recurrent colorectal cancer: Comparison with integrated FDG PET/non-contrast-enhanced CT and enhanced CT. Eur J Med Mol Imaging 36:1388-1396

5. Bailey C, Hu CY, You N et al (2015) Variation in positron emission tomography use after colon cancer resection. J Oncol Practice 11:1

6. Yu T, Meng N, Chi D et al (2015) Diagnostic value of 18F-FDG/PET CT in detecting local recurrent colorectal cancer: a pooled analysis of 26 individual studies. Cell Biochem Biophy 72:443-451

7. Kyoto Y, Momose M, Kondo C et al (2010) Ability of 18F-FDG PET/CT to diagnose recurrent colorectal cancer in patients with elevated CEA concentrations. Ann Nuclear Med 24:395-401

8. Laurens ST, Oyen WJ (2015) Impact of flurodeoxyglucose PET/computed tomography on the management of patients with colorectal cancer. PET Clinics 10:345-360

9. Agarwal A, Marcus C, Xiao J et al (2014) FDG PET/CT in the management of colorectal and anal cancers. Am J Roentgenol 203:1109-1119

10. Metser U, You J, McSweeney S et al (2010) Assessment of tumor recurrence in patients with colorectal cancer and elevated carcinoembryonic antigen level: FDG PET/CT versus contrast-enhanced 64-MDCT of the chest and abdomen. Am J Roentgenol 194:766-771 
11. Kitajima K, Nakajo M, Kaida $\mathrm{H}$ et al (2017) Present and future roles of FDGPET/CT imaging in the management of gastrointestinal cancer: an update. Nagoya J Med Sci 79:527-543

12. Chiewvit S, Apisarnthanarak P, Kanchanapiboon B et al (2013) Detection of recurrent colorectal cancer by 18 F-FDGPET/CT comparison with contrast enhanced CT scan. J Med Assoc Thai 96:703-708

13. D'Souza MM, Sharma R, Mondal A et al (2009) Prospective evaluation of CECT and 18F-FDG-PET/CT in detection of hepatic metastases. Nucl Med Commun 30:117-125

14. Ali SA, Abd Elkhalek YI (2016) Value of integrated PET/CT in detection of hepatic metastatic deposits. Egypt J Radiol Nuclear Med 47:459-465

15. Chen LB, Tong JL, Song HZ et al (2007) 18F-DG PET/CT in detection of recurrence and metastasis of colorectal cancer. World J Gastroenterol 13 5025-5029

16. Veit $\mathrm{P}$, Antoch G, Stergar $\mathrm{H}$ et al (2006) Detection of residual tumor after radiofrequency ablation of liver metastasis with dual-modality PET/CT:initial results. Eur Radiol 16:80-87

17. Bullier E, Descat E, Picat MQ et al (2013) (2013): Diagnostic accuracy of (18)F-FDG PET/CT for the detection of peritoneal carcinomatosis of colorectal origins. J Cancer Res Therapy 1:46-53

18. Kwak YJ, Kim JS, Kim HJ et al (2016) Diagnostic value of FDG-PET/CT for lymph node metastasis of colorectal cancer. World J Surgery 36:1898-1905

19. Ali SA, Abd Elkhalek YI (2016) Added value of combined 18F-FDG PET/CT for detection of osseous metastases in cancer patients. Egypt J Radiol Nuclear Med 47:453-458

\section{Publisher's Note}

Springer Nature remains neutral with regard to jurisdictional claims in published maps and institutional affiliations.

\section{Submit your manuscript to a SpringerOpen ${ }^{\circ}$ journal and benefit from:}

- Convenient online submission

- Rigorous peer review

- Open access: articles freely available online

- High visibility within the field

- Retaining the copyright to your article

Submit your next manuscript at $\boldsymbol{\nabla}$ springeropen.com 